\section{$\S 24$. Summary: Theory of Magnetic Confinement}

Itoh, K.

In the 19th IAEA Fusion Energy Conference (Lyon, October 2002), approximately 100 papers on the magnetic confinement theory are reported. They constitute a substantial part of the over-all contributions in the conference (about 380 presentations). This conference covers majority of progress that has been made in the area of fusion theory after the previous IAEA Conference at Sorrento in year 2000.

The magnetic confinement plasmas have shown complex structures and dynamics as is illustrated in Fig.1. Plasma parameters vary from center to surface. In addition to this gradual change of parameters, there are distinct steep variations in several circumstances, e.g., the edge transport barrier and internal transport barrier. The topology of magnetic surfaces changes at the plasma surface. It is also modified by the spontaneous appearance of magnetic islands in the core. The temporal evolution includes the slow change as well as abrupt transitions. Papers which is related to the magnetic confinement theory try to clarify the mechanisms that govern the structure and dynamics of plasmas. At the same time, these papers intend to provide methods for control of and for improvement of confined plasmas for realizing the ignited plasmas. Issues which are covered by the theory-related papers might be arranged as follows:

1. Theory of transport phenomena

1.1 Transport-code analysis

1.2 Transport-barrier mechanism

1.3 Anomalous torque, off-diagonal elements, etc.

1.4 Finite and long range transportation

\subsubsection{Finite correlation length}

1.4.2 Self-organized criticality (SOC) model

1.4.3 Alternative modeling based on variational principle

1.5 Large-orbit neoclassical transport theory

1.6 Linear instabilities

1.6.1 Microscopic instabilities

1.6.2 Ballooning modes

2. Direct simulation and turbulent transport

2.1 Progress of code and benchmarking
2.2 Multiple scale turbulence

2.3 Global simulation and Bohm diffusion vs gyro-Bohm diffusion

2.4 Mesoscales

2.5 Modelling helical plasmas

3. MHD models: Understanding and control

3.1 Global understanding including plasma shapes

3.2 Fast events, nonlinear instabilities

3.3 Helical systems and others

3.4 Control

4. Energetic particles and Alfven eigenmodes

4.1 Role of plasma geometry

4.2 Combined analysis of large-scale dynamics of waves and particles

4.3 Runaway electrons and ions

5. Heating and current drive

6. Edge modelling

6.1 Transport analysis with atomic and molecular processes

6.2 Other important phenomena

6.3 ELMs and pedestal

7. Statistical approaches

8. Summary and prospect

This summary is prepared to highlight the new progresses that have impacts on future research. The view point of this summary is chosen, putting an emphasis upon interactions between dynamics with different scale lengths and/or time scales. This conference covers majority of progress that has been made in the area of fusion theory. This summary paper is made by putting an emphasis on aspects as follows. First, the integration is the area where many efforts were made. Many models have been developed in the history of theory of magnetic confinement plasmas, based on the assumption of scale separation. Combination of dynamics that are describing various processes with different scales provides the new dynamics and structures. Thus new findings are made through integration of models. This is because the confined plasma is in the far-nonequilibrium state with strong nonlinearity and the integrated system is far away from the linear superposition of parts. In addition to this integration, the importance of the mutual interaction between different scales is also highlighted in this conference, and is emphasized in this summary. The study of the mutual interactions between different scales, before closing a model, is one of the main outcome of this conference. 